\title{
Added value of arterial spin-labeling (ASL) and susceptibility weighted-images (SWI) in detecting a mild symptomatic intracranial arteriovenous fistula
}

\section{A importância das técnicas arterial spin-labeling e susceptibility weighted-images na} detecção de fistulas arteriovenosas

Felipe T. Pacheco 1,2, Bruna Bianca Allegro ${ }^{1,2}$, Igor Gomes Padilha ${ }^{1,2}$, Renato Hoffmann Nunes ${ }^{1,2}$, Antônio José da Rocha ${ }^{1,2}$

Dural arteriovenous fistulas consist of a direct arteriovenous shunt, across the dural layer, without an intervening capillary nidus. An intracranial dural arteriovenous fistula diagnosis remains challenging as symptoms vary according to their location ${ }^{1}$.

A 26-year-old woman presented with left pulsatile tinnitus one year after an intracranial venous thrombosis. Conventional brain MRI sequences were unremarkable. However, arterial spinlabeling and susceptibility weighted imaging suggested blood flow abnormalities in the left posterolateral tentorial region (Figure), supporting suspicion of a dural arteriovenous fistula. This diagnosis was confirmed by magnetic resonance angiography, allowing appropriate treatment. This report reinforces the importance of these new techniques on high magnetic field MRI to confidently reveal some vascular disorders that have remained obscured until recently. It is also important to highlight the advantage that both techniques do not require contrast administration ${ }^{2}$.
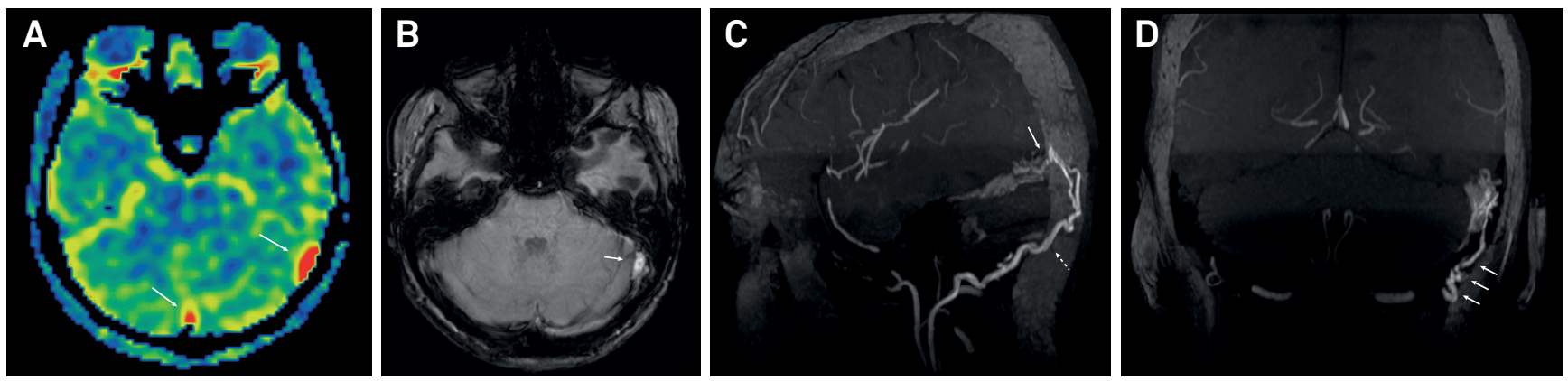

Figure. (A) Abnormal signal intensities due to oxygenated blood in arterial reflux into venous system (transverse and sagittal superior sinuses depicted on arterial spin-labeling perfusion - arrows) and (B) axial susceptibility weighted image (arrow). Sagittal maximum intensity projection (C) identified prominent left occipital artery (dotted arrow) and the connections between small adjacent transosseous branches and dural sinus, confirming a dural arteriovenous fistula (arrow). Coronal magnetic resonance angiography using maximum intensity projection (D) showed numerous extradural arteries converging to the posterolateral petrotentorial region (arrows).

1. Reynolds MR, Lanzino G, Zipfel GJ.

Intracranial dural arteriovenous fistulae.

Stroke. 2017;48(5):1424-31.

https://doi.org/10.1161/STROKEAHA.116.012784
2. Hodel J, Leclerc X, Kalsoum E, Zuber M, Tamazyan R, Benadjaoud MA et al. Intracranial arteriovenous shunting: detection with arterial spinlabeling and susceptibility-weighted imaging combined. AJNR Am J Neuroradiol. 2017 Jan;38(1):71-6. https://doi.org/10.3174/ajnr.A4961

\footnotetext{
${ }^{1}$ Faculdade de Medicina da Santa Casa de São Paulo, Divisão de Neurorradiologia, São Paulo SP, Brasil;

${ }^{2}$ Diagnósticos da América, São Paulo SP, Brasil.

Correspondence: Felipe T. Pacheco; Rua Doutor Cesário Motta Junior 112; 01221-020 São Paulo SP, Brasil. E-mail: felipetorrespacheco@hotmail.com Author Contributions: Dra. Allegro, Dr. Padilha and Dr. Nunes: data collection and drafting the manuscript; Dr. Pacheco and Dr. Rocha: study concept and revising the manuscript.

Conflict of interest: There is no conflict of interest to declare.

Received 18 February 2018; Received in final form 19 March 2018, Accepted 31 March 2018.
} 